Ann. Génét. Sél. anim., I972, 4 (2), 281-295.

\title{
DONNÉES COMPLÉMENTAIRES SUR LES BESOINS NUTRITIONNELS DE LA REPRODUCTRICE NAINE VEDETTE I. N. R. A. JV 15
}

\author{
J. GUILLAUME \\ avec la collaboration technique de Liliane DUPUY et Joëlle BRUÈRE
}

Station de Recherches avicoles, Centre de Recherches de Tours, I. N. R. A., 37 - Nouzilly

\section{RÉSUMÉ}

Nous effectuons une expérience en vue de déterminer les besoins azoté et énergétique de la reproductrice nanifiée du type chair Vedette $I$. N.R.A.JV $15(d w) .576$ animaux provenant d'un élevage commercial, élevés en cage sont utilisés. Ils ont été rationnés à partir de ro semaines et pèsent $1,69 \mathrm{~kg}$ à $\mathrm{I} 7$ semaines; à cet âge ils sont mis en expérience. Nous utilisons 3 régimes isoénergétiques $(2,75 \mathrm{kcal} \mathrm{EM} / \mathrm{g})$ apportant I $_{5} 5^{-\mathrm{I}_{4}}$ ou I $2,5 \mathrm{p}$. Ioo de protéines (contenant 4,5 p. roo de lysine et $3,85 \mathrm{p}$. Ioo d'acides aminés soufrés). Les animaux reçoivent individuellement soit I $20 \mathrm{~g}$ d'aliment par jour, soit (lots "rationnés ") une quantité décroissante au cours de la saison de ponte. Les animaux sont en outre divisés en 4 groupes de poids.

Taux azoté et restriction alimentaire influencent l'intensité de ponte, le poids des cufs, le poids des poules et la mortalité. L'efficacité alimentaire est améliorée par le taux azoté tandis que le pourcentage d'œufs fêlés ou hardés est plus faible chez les animaux restreints.

Les animaux légers ont une intensité de ponte plus grande ; ils produisent moins d'œufs fêlés ou hardés et consomment moins, mais leur mortalité est plus élevée.

Cependant, les effets de la restriction d'énergie et de protéines sont différents : la restriction énergétique diminue légèrement le taux de ponte, fortement le gain de poids et améliore l'efficacité alimentaire. Elle touche surtout les poules lourdes. La restriction de protéines diminue le nombre et le poids des œufs, le gain de poids et ne modifie pas l'efficacité alimentaire. Elle touche surtout les poules légères. Les effets des deux restrictions sont additifs.

Les besoins journaliers de la reproductrice $J V 15$, en valeur absolue, sont faibles : environ $285 \mathrm{kcal}$ EM et $\mathrm{I} 7 \mathrm{~g}$ de protéines (dont $0,77 \mathrm{~g}$ de lysine et $0,64 \mathrm{~g}$ d'acides aminés soufrés). Ces valeurs correspondent à un rapport protéines/énergie assez élevé, comme chez la Leghorn dw. Ce rapport élevé est peut-être dû à un besoin important en acides aminés soufrés. Dans un second essai, on trouve en effet qu'un supplément de ceux-ci peut améliorer poids de l'œuf, poids de l'albumen et croissance de la descendance.

Cet effet maternel, de même que le rôle propre du gène $d w$, est cependant à confirmer. 


\section{INTRODUCTION}

Les effets pléiotropiques du gène de nanisme récessif lié au sexe dw (HUTT, I953) sont multiples (MÉRAT, I969; MÉRAT et GUILLAUME, I969 ; JAAP et MOHAMAdian, I969; Summers, RAJARATNAM et Moran, I970). Ce gène modifierait en particulier les besoins nutritionnels de la poulette en croissance (GUILLAUME, I97I). En revanche, son influence éventuelle sur les besoins nutritionnels de la poule en ponte reste obscure : ARSCOTT et BERNIER (I968) trouvent qu'il augmente nettement le besoin protéique chez la Leghorn; GUILLAUME, LECLERCQ et BLUM (I970) ne retrouvent pas ces résultats chez une poule également nanifiée mais de taille beaucoup plus élevée : la reproductrice de type "chair "Vedette I. N.R.A.JV 15 (Selaf, France). Damron et Harms (r970) trouvent chez la Leghorn dw un besoin en acides aminés soufrés relativement élevé. Nous effectuons une nouvelle expérience sur des animaux du même croisement $J V 15$ dans le but de préciser ses besoins de ponte en protéines et en énergie.

\section{MATÉRIEL ET MÉTHODES}

\section{Expérience I}

Cette expérience porte sur 576 poules provenant d'un élevage commercial. Ces animaux n'ayant été rationnés qu'à partir de l'âge de Io semaines, pèsent I, 7 kg à I 7 semaines ; à cet âge-ci ils sont mis dans les cages de ponte expérimentales.

Les animaux sont divisés en 4 groupes formés d'animaux de poids comparables et, à l'intérieur de chaque groupe, ils sont répartis au hasard entre les 6 traitements alimentaires éprouvés. Chacune des 24 combinaisons traitement $\times$ groupe de poids est représentée 2 fois à chaque étage des batteries ( 2 blocs par étage) et la répartition à l'intérieur de chacun de ces blocs est faite au hasard.

La composition des 3 régimes éprouvés figure dans le tableau I. Il s'agit de 3 régimes isoénergétiques de taux variables en protéines. Les teneurs de la protéine brute en lysine et acides aminés soufrés sont maintenues constantes : 4,4 et 3,8 p. Ioo respectivement. Le taux azoté voisin du besoin d'une pondeuse classique dans le régime $\mathrm{A}$ est inférieur de ıo $\mathrm{p}$. Ioo dans le régime $\mathrm{B}$ et de $20 \mathrm{p}$. 100 dans le régime $\mathrm{C}$.

Tous les animaux sont nourris individuellement, recevant 3 fois par semaine une ration pesée. Une série de 3 lots reçoit la même dose du début de la ponte à la fin de l'essai (de 22 à 62 semaines). Les lots sont désignés par la lettre servant à caractériser l'aliment : A, B et C. Les 3 autres lots subissent d'abord un rationnement comparable puis, une fois le pic de ponte passé, un rationnement de plus en plus sévère (fig. I). Ces lots sont désignés par les symboles AR, BR et CR.

Durant tout l'essai on enregistre pour chaque poule, la consommation, le nombre et le poids des œufs ainsi que le nombre d'œufs hardés ou fêlés et d'œufs à double jaune. La mortalité et ses causes sont également enregistrées.

On effectue l'interprétation statistique des résultats par une double analyse de variance : la première porte sur le dispositif factoriel $3 \times 2$ (régime $\times$ rationnement) sans tenir compte du poids des animaux, la seconde sur le dispositif factoriel $6 \times 4$ (combinaisons régime-rationnement $\times$ classe de poids). Afin de rendre l'analyse possible malgré l'inégalité du nombre d'animaux dans chaque lot (par suite de la mortalité), on fait appel à la technique des "fittings constants".

Nous avons complété l'analyse statistique par des calculs de régression multiple entre la production individuelle en poids d'œufs pondus (variable expliquée) et la consommation de protéines et d'énergie (variables explicatives). La technique choisie a été celle de la "régression progressive par choix $"$. 
TABLEAU $\mathbf{I}$

Composition des régimes expérimentaux

\begin{tabular}{|c|c|c|c|}
\hline & \multicolumn{3}{|c|}{ Régimes } \\
\hline & 1 & 2 & 3 \\
\hline \multicolumn{4}{|l|}{ Composants } \\
\hline Avoine $\ldots \ldots \ldots \ldots \ldots \ldots \ldots \ldots \ldots \ldots \ldots$ & 15,8 & 10,8 & 6,5 \\
\hline 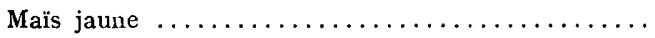 & 54,6 & 60,0 & 64,8 \\
\hline Farine de luzerne déshydratée $(17 \%) \ldots \ldots \ldots \ldots \ldots$ & 4,0 & 4,0 & 5,0 \\
\hline Tourteau de soya $50 \% \ldots \ldots \ldots \ldots \ldots \ldots$ & 5,6 & 3,0 & 1,7 \\
\hline Tourteau de tournesol $42-44 \% \ldots \ldots \ldots \ldots \ldots$ & 5,0 & 3,6 & 0 \\
\hline Farine de poisson de Norvège $70 \% \ldots \ldots \ldots \ldots$ & 1,0 & 1,0 & 1,0 \\
\hline Farine de viande $50 \% \ldots \ldots \ldots \ldots \ldots \ldots$ & 5,0 & 5,0 & 5,0 \\
\hline 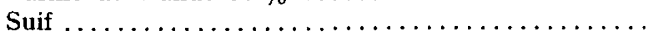 & 1,0 & 1,0 & 1,0 \\
\hline 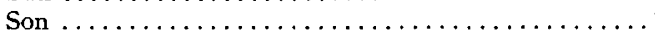 & 0 & 3,6 & 7,0 \\
\hline DL-méthionine $\ldots \ldots \ldots \ldots \ldots \ldots \ldots \ldots \ldots$ & 0,025 & 0 & 0 \\
\hline Carbonate de $\mathrm{Ca}$ (Calcimarine) $\ldots \ldots \ldots \ldots \ldots \ldots$ & 6,225 & 6,25 & 6,25 \\
\hline Bicarbonate de sodium $\ldots \ldots \ldots \ldots \ldots \ldots \ldots$ & 0,6 & 0,6 & 0,6 \\
\hline 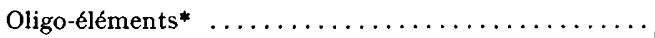 & 0,15 & 0,15 & 0,15 \\
\hline Prémélange vitaminique $^{* *} \ldots \ldots \ldots \ldots \ldots \ldots \ldots$ & 1,0 & 1,0 & 1,0 \\
\hline \multicolumn{4}{|l|}{ Analyse } \\
\hline 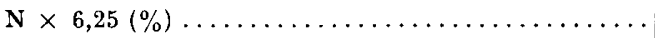 & 15,6 & 14 & 12,5 \\
\hline 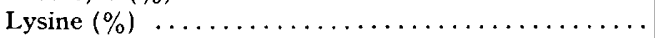 & 0,70 & 0,60 & 0,52 \\
\hline 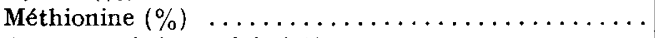 & 0,30 & 0,25 & 0,22 \\
\hline Acides aminés soufrés $(\%) \ldots \ldots \ldots \ldots \ldots \ldots$ & 0,60 & 0,52 & 0,45 \\
\hline 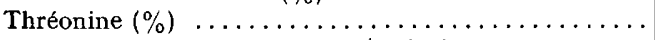 & 0,56 & 0,50 & 0,43 \\
\hline Énergie métabolisable $\mathrm{kcal} / \mathrm{g}$ \{ calculée $\ldots \ldots \ldots \ldots$ & 2,75 & 2,75 & 2,75 \\
\hline Energie métabolisable $\mathrm{kcal} / \mathrm{g}$ mesurée $\ldots \ldots \ldots \ldots \ldots$ & 2,82 & 2,75 & 2,78 \\
\hline Matières grasses $(\%) \ldots \ldots \ldots \ldots \ldots \ldots \ldots$ & 5,1 & 5,15 & 5,2 \\
\hline Cellulose brute $(\%) \ldots \ldots \ldots \ldots \ldots \ldots \ldots \ldots$ & 4,8 & 4,6 & 4,4 \\
\hline 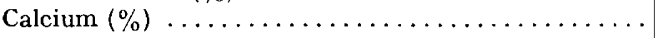 & 3,1 & 3,1 & 3,1 \\
\hline Phosphore disponible $(\%) \ldots \ldots \ldots \ldots \ldots \ldots$ & 0,4 & 0,4 & 0,4 \\
\hline
\end{tabular}

* Apportant en g pour $100 \mathrm{~kg}$ : Sulfate de $\mathrm{Cu}, 5 \mathrm{H}_{2} \mathrm{O}=0,315$; Sulfate de $\mathrm{Co}, 7 \mathrm{H}_{2} \mathrm{O}=0,045$; Molybdate de $\mathrm{Na}=0,0225$; Sulfate de $\mathrm{Zn}, 7 \mathrm{H}_{2} \mathrm{O}=6,75$; Iodure de $\mathrm{K}=0,0675$; Carbonate de Ca $=15,075 ;$ Fucus $=2,25 ;$ Sulfate de $\mathrm{Mn}, 4 \mathrm{H}_{2} \mathrm{O}=9,0 ;$ Oxyde de $\mathrm{Mg}, 7 \mathrm{H}_{2} \mathrm{O}=4,5 ;$ Carbonate de $\mathrm{Mg}=3,6$; Sulfate de $\mathrm{Fe}, 7 \mathrm{H}_{2} \mathrm{O}=3,375$.

** Apportant pour $100 \mathrm{~kg}$ : Vitamine $\mathrm{A}=800000 \mathrm{UI}$; Vitamine $\mathrm{D}_{3}=100000 \mathrm{UI}$; Tocophérol $=3 \mathrm{~g}$; Riboflavine $=0,5 \mathrm{~g} ;$ Nicotinamide $=1 \mathrm{~g} ;$ Vitamine $\mathrm{B}_{12}=0,2 \mathrm{mg} ;$ Choline $=12,5 \mathrm{~g} ; \mathrm{BHT}=15 \mathrm{~g}$; Avoine $=$ q.s.p.

\section{Expérience 2}

Cette expérience a pour but de vérifier l'existence d'un " effet maternel » d'origine alimentaire chez des descendants de poules $J V 15$ : Dans un essai antérieur, nous avions constaté que la supplémentation avec $0,22 \mathrm{p}$. I 100 de lysine et $0,16 \mathrm{p}$. Ioo de DL-méthionine d'un aliment contenant 2,8 kcal EM/g, I6, I5 p. Ioo de protéines, o,66 p. roo de lysine et o,57 p. roo d'acides aminés soufrés avait pour seul effet une augmentation de $5 \mathrm{p}$. Ioo environ du poids à 4 semaines de la descendance (Guillaume, données non publiées).

Dans l'expérience 2, nous cherchons à identifier l'acide aminé auquel est due cette amélioration. Pour cela nous utilisons 48 poules issues du même troupeau que dans l'expérience 1 et élevées également en cages individuelles. Elles sont nourries de l'aliment $\mathbf{B}$, durant une première période de 6 semaines à la fin de laquelle on mesure le poids de l'œuf, de l'albumen et du vitellus 


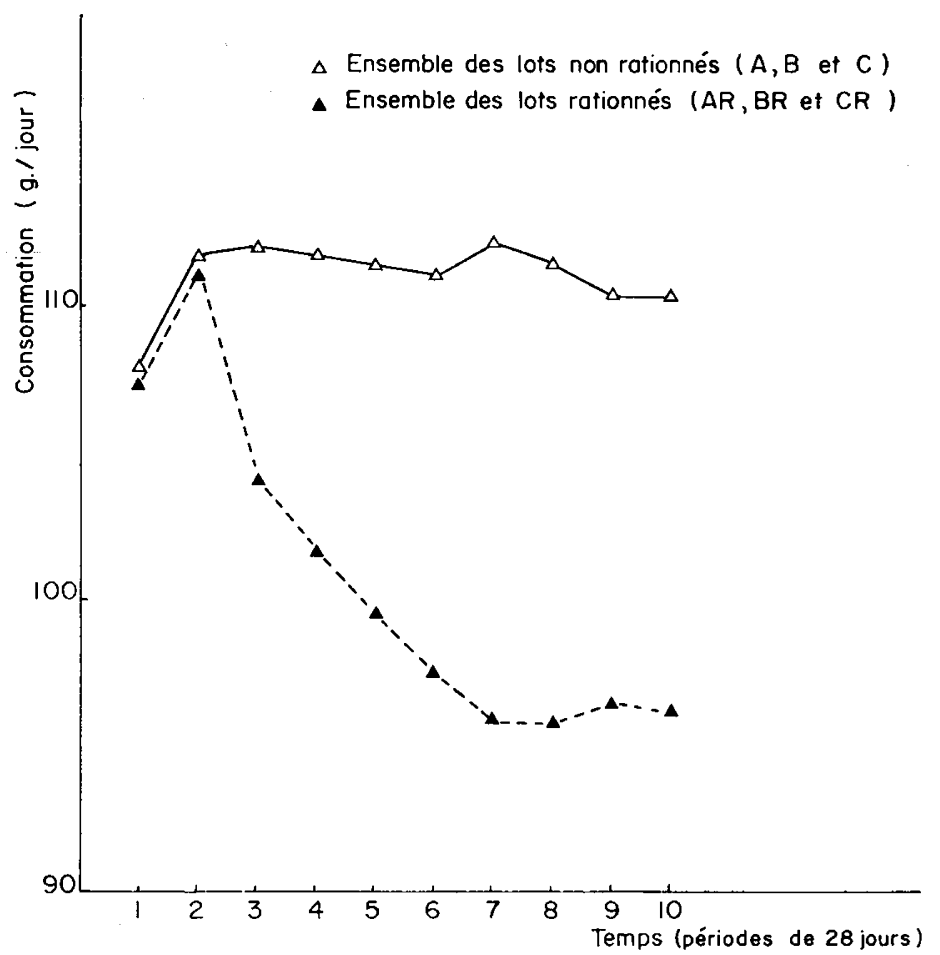

FIG. I. - Évolution du rationnement imposé aux animaux au cours du temps

TABLEAU 2

Schéma de l'expérience 2

\begin{tabular}{|c|c|c|c|c|}
\hline Périodes & $\begin{array}{c}\text { Durée } \\
\text { (semaines) }\end{array}$ & Régimes & Critères mesurés & Critères analysés \\
\hline Préexpérience & 6 & $\mathrm{~B}$ & $\begin{array}{l}\text { Poids de l'œuf entier }(\mathrm{O}) \\
\text { du vitellus }(\mathrm{V}) \\
\text { et de l'albumen }(\mathrm{A}) \\
\text { Poids des descendants } \\
\text { à } 2-4-6 \text { et } 8 \text { semaines } \\
\mathrm{P}^{2}, \mathrm{P}^{4}, \mathrm{P}^{6} \text {, et } \mathrm{P}^{8}\end{array}$ & $\begin{array}{c}\text { Moyennes } \\
\text { individuelles de } \\
\mathrm{O}_{2}-\mathrm{O} \\
\mathrm{V}_{2}-\mathrm{V}\end{array}$ \\
\hline $\begin{array}{l}\text { Expérience } \\
\text { proprement } \\
\text { dite }\end{array}$ & 6 & $\begin{array}{l}\text { Selon les lots } \\
\mathrm{B} \\
\mathrm{B}+0,2 \% \text { L-lysine } \\
\mathrm{B}+0,2 \% \text { DL-méthionine } \\
\mathrm{B}+0,2 \% \text { L-lysine } \\
+0,2 \% \text { DL-méthionine }\end{array}$ & $\begin{array}{c}\text { Poids de l'œuf entier }\left(\mathrm{O}_{2}\right) \\
\text { du vitellus }\left(\mathrm{V}_{2}\right) \\
\text { et de l'albumen }\left(\mathrm{A}_{2}\right) \\
\text { Poids des descendants } \\
\text { à } 2-4-6 \text { et } 8 \text { semaines } \\
\left(\mathrm{P}_{2}^{2}, \mathrm{P}_{2}^{4}, \mathrm{P}_{2}^{6}, \mathrm{P}_{2}^{8}\right)\end{array}$ & $\begin{array}{l}\mathrm{P}_{2}^{2}-\mathrm{P}^{2} \\
\mathrm{P}_{2}^{4}-\mathrm{P}^{4} \\
\mathrm{P}_{6}^{2}-\mathrm{P}^{6} \\
\mathrm{P}_{2}^{8}-\mathrm{P}^{8}\end{array}$ \\
\hline
\end{tabular}


de chaque poule. On insémine artificiellement les pondeuses et on élève les descendants - pédigrés - jusqu'à 8 semaines avec un aliment commercial. Durant une deuxième période, les animaux sont répartis en 4 lots de $\mathrm{I} 2$, recevant, toujours avec rationnement, soit le régime $\mathrm{B}$, soit ce régime supplémenté en lysine, méthionine et méthionine + lysine (tabl. r). Les mêmes mesures sont faites sur les œufs et un certain nombre de descendants des mêmes poules et mêmes coqs est à nouveau élevé au sol. On compare ensuite pour chaque animal une grandeur donnée (poids de l'œuf, de l'albumen ou des descendants d'âge donné) déterminée durant la deuxième période à la même grandeur déterminée durant la première période selon la méthode mise en œuvre par ailleurs par LECLERCQ, 1968.

\section{RÉSULTATS}

\section{Expérience 1}

Les principaux résultats de l'expérience I figurent dans les tableaux 3 et 4 . I1 est clair que les 2 facteurs éprouvés, niveau azoté et sévérité du rationnement, ont une incidence directe sur le nombre d'œufs pondus et le poids moyen de l'œuf. I a production est d'autant meilleure que le régime est plus concentré en protéines et qu'il est alloué plus abondamment.

\section{TABLEAU 3}

Résultats généraux de l'expérience 1 Effets du végime et du rationnement

\begin{tabular}{|c|c|c|c|c|c|c|}
\hline & A & AR & $\mathrm{B}$ & $\mathrm{BR}$ & $\mathrm{C}$ & $\mathrm{CR}$ \\
\hline Consommation $(g / j) \ldots \ldots \ldots$ & 111,4 & 101,2 & 110,9 & 101,0 & 111,5 & 99,7 \\
\hline Nombre d'œufs pondus....... & 141,1 & 131,7 & 135,7 & 123,4 & 131,7 & 116,6 \\
\hline Taux de ponte $(\%) \ldots \ldots \ldots$ & 50,4 & 47,0 & 48,5 & 44,1 & 47,0 & 41,6 \\
\hline Poids moyen de l'ouf ....... & 59,3 & 58,2 & 57,8 & 57,6 & 57,0 & 55,3 \\
\hline Consommation par œuf $(\mathrm{g}) . .$. & 220,9 & 215,1 & 228,8 & 229,2 & 237,1 & 239,4 \\
\hline Consommation par g d'œuf $(\mathrm{g}) .$. & 3,72 & 3,69 & 3,96 & 3,98 & 4,16 & 4,33 \\
\hline Gain de poids $(g / j)\left({ }^{1}\right) \ldots \ldots \ldots$ & 3,61 & 2,83 & 3,37 & 2,70 & 2,75 & 2,43 \\
\hline Mortalité $(\%) \ldots \ldots \ldots \ldots \ldots$ & 9,4 & 6,2 & 14,6 & 4,2 & 8,3 & 16,7 \\
\hline
\end{tabular}

(1) Mesuré sur 45 semaines et non sur 40 .

La consommation par œuf, en revanche, bien que diminuée par le taux azoté, n'est pas influencée par l'intensité du rationnement.

Le gain de poids des pondeuses atteint des valeurs très élevées dans tous les lots puisque, à la fin de l'essai, il équivaut à $50 \mathrm{p}$. Ioo du poids à I7 semaines. Il est lui aussi influencé directement et par le taux protidique et par la quantité d'aliment ingérée. Tous les effets mentionnés ci-dessus sont hautement significatifs $(p<0$,or.)

Sans qu'il soit possible de tirer une conclusion sûre, on doit signaler une influence apparente des facteurs éprouvés sur la mortalité qui est réduite par le rationnement sauf dans le cas de l'aliment le plus pauvre en protéines. Un autre résultat inattendu du rationnement est la réduction de la fréquence des œufs fêlés ou hardés ( 17 p. IOo). 
Le poids des poules à l'entrée en ponte n'est pas sans effets sur les performances : les poules légères mangent un peu moins (malgré une limitation identique, c'est-à-dire grâce à des refus plus importants) et pondent davantage (effets significatifs $p<0,05)$. Leur efficacité alimentaire est très nettement supérieure à celle des animaux plus lourds et le nombre d'œufs fêlés ou hardés est deux fois plus faible que chez les animaux lourds, tandis que le poids moyen des œufs ne diffère pas significativement d'un lot à l'autre. On peut également mentionner que les poules les plus légères gagnent davantage de poids et que leur mortalité est plus élevée.

La figure 2 illustre la production moyenne des lots A, B, C et AR, BR, CR réunis ainsi que celle des 2 groupes de production extrême groupe de poids $\mathrm{I}$, traitement A et groupe de poids 4 , traitement $\mathrm{CR}$.

Aucune interaction du premier ordre traitement $\times$ groupe de poids ou régime $\times$ rationnement ne s'avère significative. Les interactions régime $\times$ rationnement $\times$ groupe de poids n'ont pas été testées $\left({ }^{1}\right)$. Nous les avons cependant examinées en étudiant sur différents groupes de poids, les effets propres d'une restriction de protéines avec apport énergétique inchangé (B comparé à $A$ ), d'une restriction de 1'énergie avec apport azoté inchangé ( $A R$ comparé à $B$ ) et d'une restriction simultanée de l'énergie et des protéines (AR comparé à A) (tabl. 5).

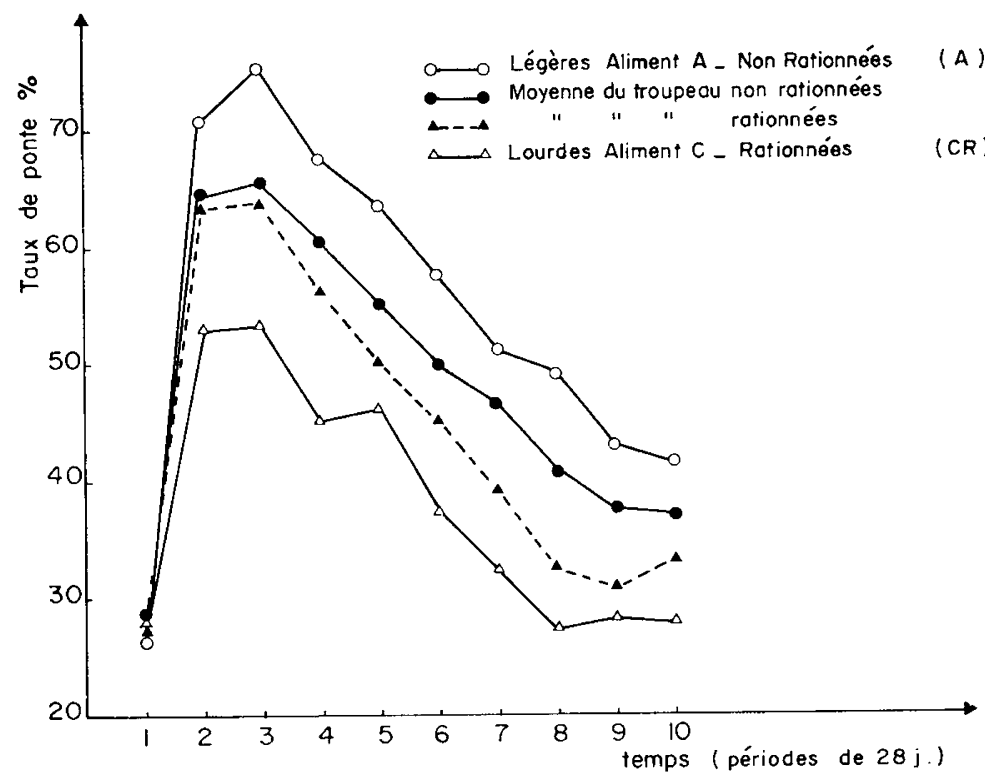

Fig. 2. - Courbe de ponte d'animaux de lots extrêmes

La courbe de ponte des animaux légers du lot A peut être considérée comme normale pour le croisement

Le rationnement en énergie n'a qu'un seul et léger effet néfaste sur la production de l'ensemble du troupeau : il réduit la ponte de $2,5 \mathrm{p}$. Ioo. En revanche, il ne touche pas le poids moyen de l'œuf et améliore même quelque peu l'efficacité alimentaire exprimée en quantité d'aliment par œuf pondu ou par g d'œufs pondus. Le rationnement en protéines atteint plus sévèrement les performances : il abaisse le 
TABLEAU 4

Résultats généraux de l'expérience 1

Effets de la classe de poids

\begin{tabular}{|c|c|c|c|c|c|c|}
\hline & \multirow[b]{2}{*}{1} & \multirow[b]{2}{*}{2} & \multirow[b]{2}{*}{3} & \multirow[b]{2}{*}{4} & \multicolumn{2}{|c|}{ Interprétation statistique } \\
\hline & & & & & $\begin{array}{l}\text { Effet de la } \\
\text { classe de poids } \\
\text { (P) }\end{array}$ & $\begin{array}{c}\text { Effet } \\
\text { de l'interaction } \\
\mathrm{P} \times \mathrm{T}\left(\mathbf{(}^{\mathbf{2}}\right)\end{array}$ \\
\hline Poids initial $(\mathrm{g}) \ldots \ldots \ldots \ldots$ & 1507 & 1643 & 1713 & 1853 & - & - \\
\hline Consommation $(\mathrm{g} / \mathrm{j})$ & 102,7 & 105,8 & 106,9 & 108,3 & $\mathrm{P}<0,01$ & - \\
\hline Nombre d'œufs..... & 133,7 & 132,4 & 128,5 & 125,9 & NS $\left({ }^{3}\right)$ & NS \\
\hline Taux de ponte $(\%) \ldots \ldots$ & 47,7 & 47,3 & 45,9 & 45,0 & NS $\left({ }^{3}\right)$ & NS \\
\hline Poids moyen de l'œuf $(g) \ldots$ & 57,0 & 57,7 & 57,6 & 57,8 & NS & NS \\
\hline Consommation/œuf ........ & 215,0 & 223,7 & 233,0 & 240,9 & - & - \\
\hline Consommation/g d'œuf & 3,77 & 3,88 & 4,05 & 5,04 & - & - \\
\hline Gain de poids $g / j\left({ }^{1}\right) \ldots \ldots$ & 3,04 & 2,91 & 2,86 & 2,94 & - & - \\
\hline Mortalité $(\%) \ldots \ldots \ldots \ldots$ & 13,2 & 9,7 & 10,4 & 6,2 & & \\
\hline
\end{tabular}

(1) Mesuré sur 45 semaines et non sur 40 .

(2) $\mathrm{T}:$ traitement : régime et rationnement (tabl. 4).

( $\left.{ }^{3}\right)$ Cet effet non significatif sur l'ensemble des œufs pondus apparaît cependant hautement significatif quand on élimine les œufs fêlés et hardés. Le nombre de ces œufs est en effet beaucoup plus élevé chez les poules lourdes.

poids des œufs et plus encore leur nombre, mais il ne modifie pas l'efficacité alimentaire. Les deux rationnements limitent la prise de poids des animaux mais à ce point de vue la restriction énergétique est plus efficace ( $16 \mathrm{p}$. Ioo au lieu de $7 \mathrm{p}$. roo). Le rationnement simultané en énergie et protéines a un effet cumulatif : les performances et le gain de poids sont nettement réduits, l'efficacité alimentaire est pratiquement inchangée.

Poules légères et poules lourdes réagissent toujours de façon différente aux diverses restrictions. Dans le cas du gain de poids, les poules lourdes se montrent beaucoup plus sensibles que les légères aux deux sortes de restriction. En revanche, si l'on considère les performances et l'efficacité alimentaire, on constate une sensibilité à la restriction protéique plus grande chez des poules légères. Il semble que, à l'opposé, les performances des poules lourdes souffrent davantage de la restriction énergétique. Étant donné les variabilités des valeurs enregistrées sur les poules lourdes, il est cependant difficile d'affirmer que ces changements ont une signification. Les études de régression simple ou multiple (tabl. 6) confirment les résultats précédents : l'importance primordiale des protéines chez les poules autres que celles du groupe 4 (les plus lourdes) est illustrée par la corrélation plus élevée existant entre le poids des œufs pondus $(z)$ et les protéines ingérées $(x)$ qu'entre le poids des œufs pondus et l'énergie ingérée. L'introduction de la variable explicative $y$ (énergie ingérée) n'améliore significativement l'ajustement que dans 2 cas sur 8 . Il faut cependant noter que chez les poules lourdes, la variable la mieux reliée à la production est l'énergie ingérée.

(1) Leur calcul avec la méthode des « fittings constants " eût été des plus laborieux. 


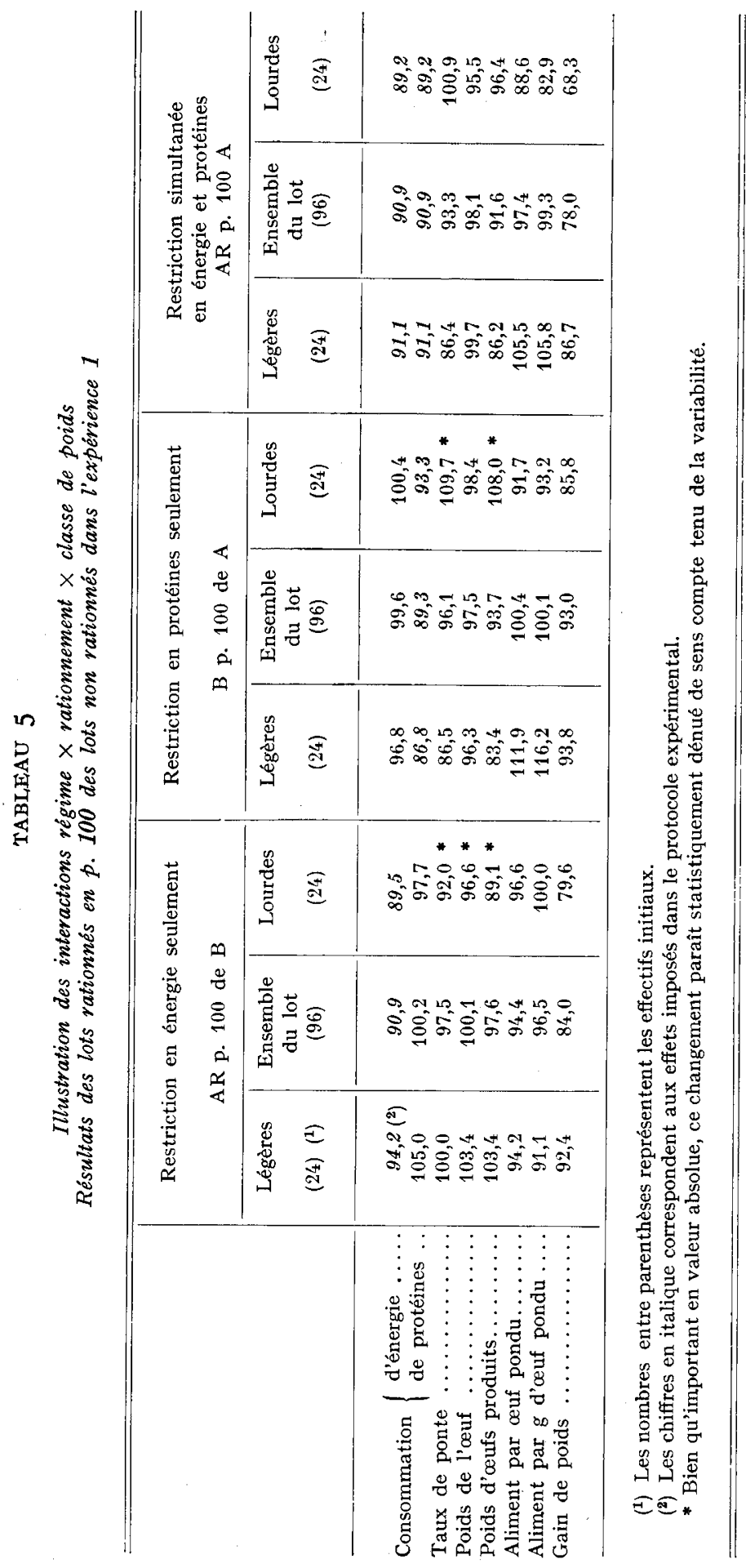




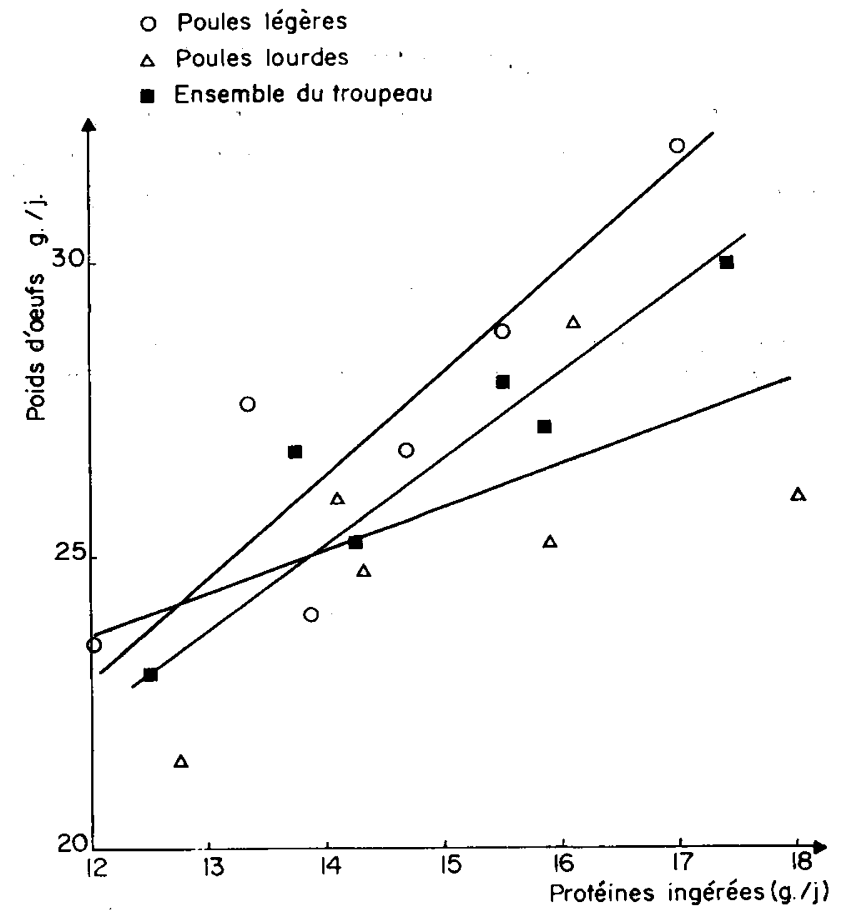

FIG. 3. - Relation entre production d'oufs (en $\mathrm{g} / \mathrm{j}$ ) et consommation d'energie et de protéines chez les animaux légers (groupe de poids I), lourds (groupe de poids 4) et sur l'ensemble du troupeau

- Poules légères

$\Delta$ Poules lourdes

- Ensembie du troupeou

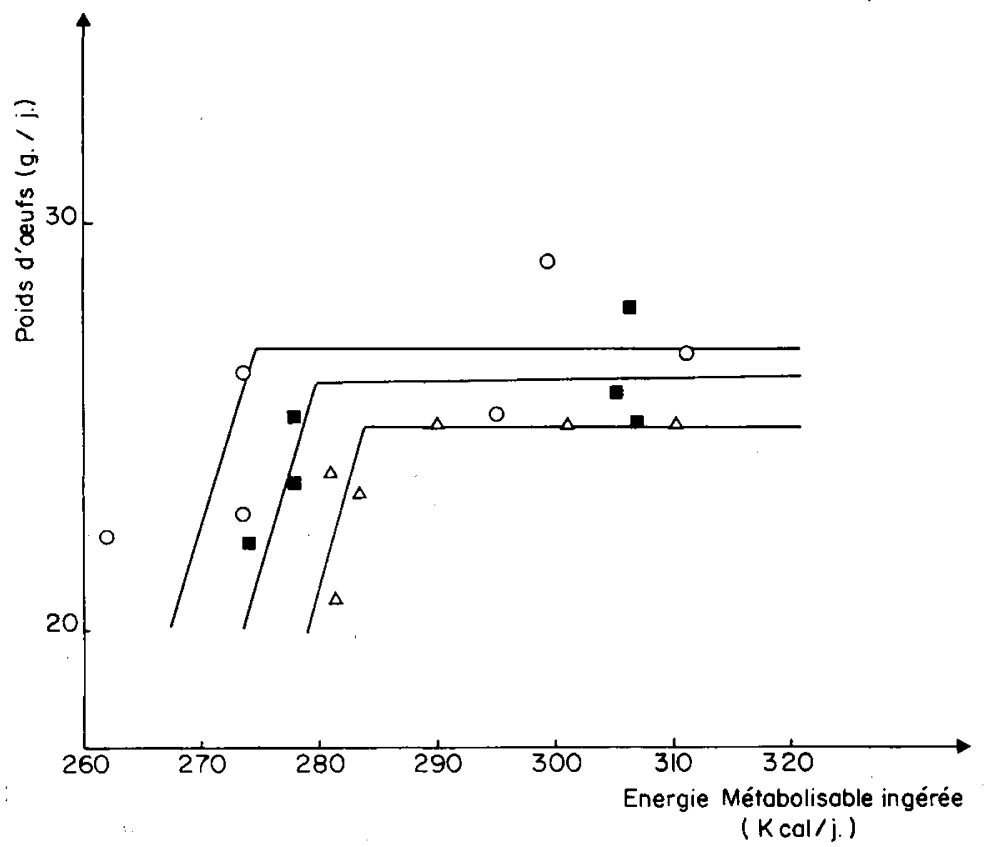

FIG. 4. - Relation entre prodution d'œufs (en $\mathrm{g} / \mathrm{j}$ ) et énergie métabolisable ingérée (kcal/j) chez les animaux legers (groupe poids I), lourds (groupe de poids 4) et sur l'ensemble du troupeau 
Nous devons aussi noter que la mauvaise corrélation existant entre production et énergie ingérée peut être attribuée à la non linéarité de la liaison. Il semble en effet que l'énergie ingérée n'améliore la ponte qu'en dessous d'une valeur bien définie (fig. 3).

\section{TABLEAU 6}

Relation entre poids des ceufs pondus (en $\mathrm{g}$ ) $=z$ d'une part et consommation de protéines $x$ (en g) et d'énergie $g$ (en kcal) d'autre part

\begin{tabular}{|c|c|c|c|c|c|c|}
\hline $\begin{array}{l}\text { Groupe } \\
\text { de poids }\end{array}$ & $\begin{array}{l}\text { Période } \\
\text { de ponte }\end{array}$ & $r_{z x}$ & $r_{z y}$ & $\mathrm{R}_{z . x y}$ & d. 1 . & Équation de régression \\
\hline \multirow{2}{*}{1 (légères) } & 3 à 4 & 0,520 & 0,494 & 0,541 & 130 & $z=1,372 x+0,072 y-0,373$ \\
\hline & 1 à 10 & 0,553 & 0,553 & 0,579 & 122 & $z=1,049 x+0,069 y-2,555$ \\
\hline \multirow{2}{*}{2} & 3 à 4 & 0,361 & 0,227 & $(0,362)$ & 137 & $\begin{aligned} z & =1,630 x-0,469 \\
(z & =1,749 x-0,014 y+0,592)\end{aligned}$ \\
\hline & 1 à 10 & 0,294 & 0,183 & $(0,294)$ & 127 & $\begin{aligned} z & =1,247 x+1,625 \\
(z & =1,209 x+0,005 x+1,341)\end{aligned}$ \\
\hline \multirow[t]{2}{*}{3} & 3 à 4 & 0,441 & 0,384 & $(0,456)$ & 136 & $\begin{aligned} z & =1,880 x+0,251 \\
(z & =1,427 x+0,052 y-0,223)\end{aligned}$ \\
\hline & 1 à 10 & 0,442 & 0,419 & 0,485 & 126 & $z=1,275 x+0,084 y-3,64$ \\
\hline \multirow{2}{*}{4 (lourdes) } & 3 à 4 & 0,239 & 0,216 & $(0,239)$ & 135 & $\begin{aligned} z & =1,367 x+0,609 \\
(z & =0,984 x+0,065 y-0,165)\end{aligned}$ \\
\hline & 1 à 10 & 0,147 & 0,180 & $(0,188)$ & 132 & $\begin{aligned} z & =0,071 y+0,54 \\
(z & =0,291 x+0,056 y+0,545)\end{aligned}$ \\
\hline
\end{tabular}

Les coefficients de corrélations multiples ( $R$ ) et des équations de régression multiple entre parenthèses correspondent aux cas où l'introduction de la seconde variable explicative n'a pas amélioré la précision de l'ajustement.

Il faut noter que les valeurs de $R$ sont anormalement basses du fait de la présence de poules n'ayant pas pondu ou ayant seulement pondu quelques œufs.

\section{Expérience 2}

Les résultats des essais non publiés mentionnés dans 1'introduction et ceux de l'expérience 2 figurent dans les tableaux 7 et 8 .

L' " effet maternel " constaté antérieurement se retrouve dans cette expérience. La supplémentation des régimes maternels en méthionine ou en méthionine et lysine améliore la croissance de la descendance femelle à 2, 4 et 6 semaines d'âge. En revanche, l'addition de lysine seule n'a aucun effet. Cette amélioration n'est significative qu'à 2 semaines d'âge : elle semble subsister jusqu'à 6 semaines d'âge en valeur absolue, mais n'est plus significative.

Il faut aussi noter que, contrairement à ce que l'on avait vérifié dans l'essai antérieur, le régime de base était carencé en acides aminés indispensables : de la période préexpérimentale à la période expérimentale le poids de l'œuf augmente plus 
TABLEAU 7

Influence du régime maternel sur la croissance des descendants de poules $J V 15$ élevés en batteries avec un aliment unique

(Guillaume, données non publiées)

\begin{tabular}{|c|c|c|c|}
\hline \multirow{2}{*}{ Régime maternel } & \multicolumn{2}{|c|}{ Poids à 4 semaines $(\mathrm{g})$} & \multirow{2}{*}{$\begin{array}{c}\begin{array}{c}\text { Indice de } \\
\text { consom- } \\
\text { mation }\end{array} \\
\begin{array}{c}\text { Sexes } \\
\text { mélangés }\end{array}\end{array}$} \\
\hline & M & $\mathrm{F}$ & \\
\hline $\begin{array}{c}2,8 \mathrm{kcal} \text { EM/g } \\
16,15 \text { p. } 100 \mathrm{~N} \times 6,25 \ldots \ldots \ldots \ldots \\
\operatorname{dont}\left\{\begin{array}{l}0,66 \mathrm{p} .100 \text { lysine } \\
0,57 \text { p. } 100 \text { acides aminés soufré }\end{array}\right.\end{array}$ & $\begin{array}{l}438,9 \\
(19)^{* *}\end{array}$ & $\begin{array}{c}410,3 \\
(16)\end{array}$ & 2,03 \\
\hline $\begin{array}{c}2,8 \mathrm{kcal} \mathrm{EM} / \mathrm{g} \\
16,3 \text { p. } 100 \mathrm{~N} \times 6,25 \ldots \ldots \ldots \ldots \\
\text { dont }\left\{\begin{array}{l}0,88 \mathrm{p} .100 \text { lysine } \\
0,73 \mathrm{p} .100 \text { acides aminés soufré }\end{array}\right.\end{array}$ & $\begin{array}{c}472,1 \\
(19)\end{array}$ & $\begin{array}{c}428,1 \\
(16)\end{array}$ & 1,96 \\
\hline
\end{tabular}

Différence entre régimes maternels $\mathbf{P}<0,05$

* Détail de la composition donnée par Guillaume, Leclerce et BLum (1970).

** Nombre de descendants.

\section{TABLEAU 8}

Effet du régime maternel sur la croissance des descendants femelles

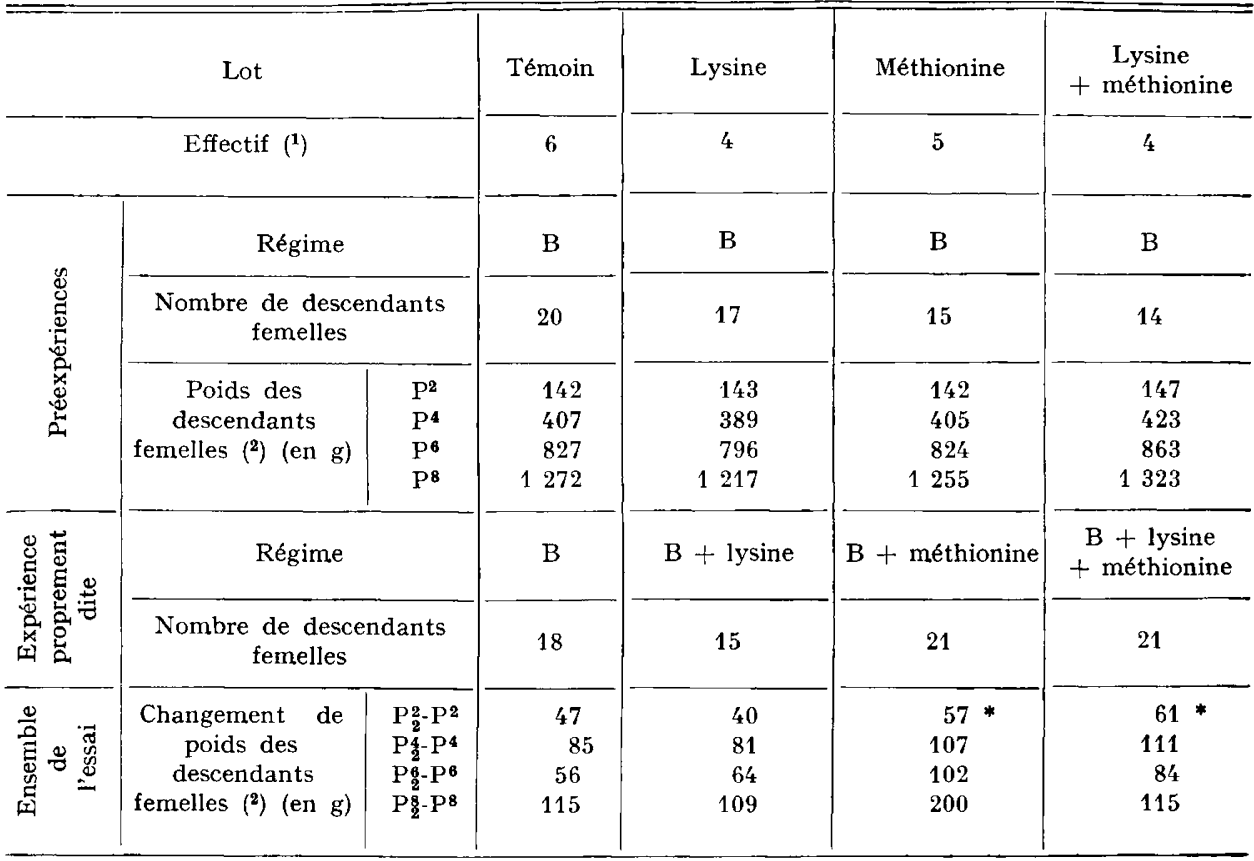

(1) Nombre d'animaux ayant donné suffisamment de descendants pour être retenus.

(2) Pour l'explication des abréviations, voir tableau 2.

* Valeurs significativement supérieures aux valeurs de 47 et 40 des lots " témoin " et "lysine " $(\mathrm{P}<0,05)$. 
vite si 1'aliment expérimental est supplémenté en lysine et surtout en méthionine (tab1. 9). L'effet bénéfique de ce dernier acide aminé porte essentiellement sur le poids de l'albumen, ce qui est en accord avec les travaux antérieurs de MoraN (I969), LECI,ERCQ (I970) et LARBIER, BLUM et GuILLAUME (I97I). L'amélioration de la croissance de la descendance suit donc une augmentation du poids de l'œuf.

TABLEAU 9

Effet du régime des poules reproductrices sur le poids de l'œuf et de ses constituants (en g) Expérience 2

\begin{tabular}{|c|c|c|c|c|c|}
\hline \multicolumn{2}{|c|}{ Effectifs des différents lots } & 6 & 4 & 5 & 4 \\
\hline \multirow{2}{*}{ 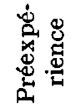 } & Régime & B & B & B & $\mathrm{B}$ \\
\hline & Poids moyen de l'œuf $\mathrm{O}$ & 53,5 & 55,3 & 55,2 & 57,0 \\
\hline \multirow{2}{*}{ 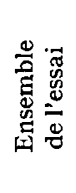 } & Régime & B & $\mathrm{B}+$ lysine & $\mathrm{B}+$ méthionine & $\begin{array}{c}B+\text { lysine } \\
+ \text { méthionine }\end{array}$ \\
\hline & $\begin{array}{ll}\text { Changements } & \mathrm{O}_{2}-\mathrm{O} \\
\text { de poids } & \mathrm{V}_{2}-\mathrm{V} \\
& \mathrm{A}_{2}-\mathrm{A}\end{array}$ & $\begin{array}{l}1,7 \\
0,5 \\
1,5\end{array}$ & $\begin{array}{l}2,4 \\
1,1 \\
1,4\end{array}$ & $\begin{array}{l}3,1 \\
1,3 \\
1,9\end{array}$ & $\begin{array}{l}3,1 \\
1,2 \\
2,1\end{array}$ \\
\hline
\end{tabular}

Noter que le poids de l'albumen a été obtenu par différence entre le poids de l'œuf entier d'une part, et celui du vitellus et de la coquille séchée d'autre part ; d'où une certaine imprécision sur sa détermination.

\section{DISCUSSION}

Dans un essai antérieur (GUILIAUME, LECLERCQ et BLUM, I970) nous avions montré que le besoin protéique de la $V$ edette $I . N . R . A . J V 15$ n'excédait pas $\mathrm{r} 6,5 \mathrm{~g}$ de protéine brute, dont 0,7 de lysine et 0,6 d'acides aminés soufrés pour une consommation énergétique de $295 \mathrm{kcal} \mathrm{EM} / \mathrm{j}$. Nos présents résultats apportent d'autres précisions : le besoin protéique est certainement très proche de la valeur indiquée précédemment puisque cette fois-ci on observe une diminution du poids de l'œuf et de l'intensité de ponte dès que la consommation journalière est inférieure à $\mathrm{I} 7 \mathrm{~g}$ (apportant $0,76 \mathrm{~g}$ de lysine et $0,65 \mathrm{~g}$ d'acides aminés soufrés). Il n'est certes pas possible de dire si une ration de $I 6,5 \mathrm{~g}$ a déjà entraîné une baisse de la production mais on peut affirmer que la valeur que nous avions indiquée était marginale dans les conditions de cet essai.

Le besoin énergétique, en revanche, paraît légèrement inférieur à la consommation observée par GUILLAUME, LECLERCQ et BLUM (I970) : Dans l'expérience présente, aucun effet néfaste n'est observé quand la consommation descend en dessous de $285 \mathrm{kcal} \mathrm{EM} / \mathrm{j}$ même chez les poules les plus exigeantes à ce point de vue à savoir les poules lourdes. 
On sait que les besoins, tant protéique qu'énergétique, sont susceptibles de varier en fonction de bien des facteurs : température ambiante, âge, poids et gains de poids des animaux, production (BYERLY, I94I ; CoMBS, I968). Nos présents résultats semblent illustrer l'influence des 3 derniers facteurs cités puisque les poules légères à l'entrée en ponte, qui pondent bien et continuent à gagner du poids, ont visiblement un besoin énergétique plus faible mais un besoin protéique plus élevé que les poules lourdes.

L'origine de l'hétérogénéité initiale du troupeau est inconnue. Elle peut résulter d'une différence de consommation durant la période de Io à $I 7$ semaines d'âge (pour des raisons de compétition et d'organisation sociale) ou de la présence de gènes modifiant la courbe de croissance. Il serait important de rechercher cette cause, par exemple avec des rationnements plus ou moins sévères durant la croissance. En effet, dans le cas où la première explication s'avérerait, une restriction sévère durant la croissance devrait s'accompagner d'une alimentation plus riche en protéines durant la ponte.

Pour l'ensemble du troupeau, la ration assurant la ponte maximale doit, dans nos conditions expérimentales, fournir environ $285 \mathrm{kcal}$ et $\mathrm{I} 7 \mathrm{~g}$ de protéines. Ces valeurs sont respectivement de 278 et I7 pour les animaux légers à production plus normale. Elles correspondent à un rapport Calories/Protéines de I64, rapport inférieur à celui trouvé habituellement chez une pondeuse classique (compte non tenu des marges de sécurité pour l'apport protéique). Si on estime le besoin énergétique de nos animaux à 1'aide des équations de BYERLY (I94I) ou ComBs (I968), on s'aperçoit en effet que leur besoin énergétique est en valeur absolue relativement faible (6 à $8 \mathrm{p}$. Ioo plus faible). En revanche, le besoin protéique est tout à fait comparable à celui d'une pondeuse de type Leghorn plus productive (BALLOuN et SPEERS, I969).

Comme la Leghorn dw (ARscotT et BERnIER, I968), la Vedette I. N. R. A. JV 15 demanderait donc un aliment riche en protéines relativement à l'énergie, mais ce résultat proviendrait en fait davantage d'une diminution du besoin énergétique estimé en valeur absolue. A ce point de vue, la poule $d w$ serait semblable au poussin $d w$ en croissance (GUILIAUME, I969) bien que son métabolisme basal paraisse peu modifié (GUIllaUmE, I970). Ce résultat semble ainsi en accord avec ceux de BERNIER et ARscotT (I960).

Il n'est cependant pas exclu que le gène $d w$ ne modifie pas également le besoin protéique et en particulier le besoin qualitatif de protéines. Les données de DAMroN et HARMS (I970) indiquent que chez la Leghorn naine dw le besoin en acides aminés soufrés relativement à l'énergie est plus élevé que chez la Leghorn normale. Les résultats de 1'expérience 2 montrent que la Vedette $I$. N.R. A.JV 15 semble avoir également un besoin élevé en acides aminés soufrés pour l'obtention du poids d'œufs maximal et pour la croissance optimale de sa descendance. Ce dernier résultat demande cependant confirmation sur un plus grand nombre d'individus. Il paraît en outre souhaitable de rechercher si " l'effet maternel " des acides aminés soufrés se manifeste encore si le poids maximal de l'œuf est atteint. En d'autres termes, le besoin en acides aminés soufrés est-il plus grand, quand on choisit comme critère la croissance de la descendance, que quand on se refère au taux de ponte ou au poids de l'œuf? Enfin, on ne sait si cet effet maternel est un phénomène général, s'il est plus particulier au croisement $J V 15$ ou s'il est spécifique des individus $d w$. 
En conclusion, dans nos conditions expérimentales, il paraît bien établi que le besoin énergétique de la poule $J V$ 15, en valeur absolue, est faible. La valeur assez élevée du rapport protéines/énergie nécessaire aux performances maximales, découle plus de la modicité du dénominateur que de l'importance du numérateur. Des travaux sur le rôle des acides aminés soufrés et sur les conséquences du rationnement durant la croissance restent cependant nécessaires à une bonne connaissance aussi bien des besoins nutritionnels de la Vedette $I . N . R . A . J V 15$ que des effets du gène $d w$ chez l'adulte.

Reçu pour publication en octobre 1971.

\title{
REMERCIEMENTS
}

Nous tenons à remercier bien vivement la Sélection avicole française (rue Rambaud, 17 La Rochelle) qui nous a fourni gracieusement les animaux ainsi que M. J. TRANChEFORT et Mlle M. F. Samour de l'Institut technique des Céréales et Fourrages (9I - Boigneville) qui ont effectué gracieusement les calculs statistiques.

\section{SUMMARY}

\author{
FURTHER DATA ON NUTRIENT \\ REQUIREMENTS OF THE DWARF BREEDER \\ HE,N VEDETTE I. N. R. A. J V I5
}

In a previous experiment we showed that, during production, the dwarf breeder hen Vedette I. N.R. A. JV 15 had a moderate energy requirement (about $290 \mathrm{kcal} \mathrm{ME} /$ day) and that increasing the protein level over 16 per cent did not improve its performance.

In this experiment we used $576 \mathrm{~J} . V .15$ breeder pullets obtained from a commercial hatchery flock and weighing $1.69 \mathrm{~kg}$ at $\mathrm{I} 7$ weeks of age. At this age they were allotted into four weight classes of equal number. Then an equal number from each weight class was assigned at random into each of the six experimental lots.

Three isocaloric diets $\left(275^{\circ} \mathrm{kcal} \mathrm{ME} / \mathrm{kg}\right.$ ) were tested. They contained 15.5, I4.0 or I2.5 per cent crude protein (diets $A, B$ and $C$ respectively). The levels of lysine and sulfur amino-acids were held constant at 4.5 and 3.85 per cent of crude protein respectively. The feed intake was limited to $\mathrm{r} 2 \mathrm{O} \mathrm{g} /$ day (lots $\mathrm{A}, \mathrm{B}$ and $\mathrm{C}$ ) or restricted more severely from the third month of lay. This restriction was accentuated until the sixth month (lots AR, BR and CR). The birds were kept in individual laying cages equipped with individual feeders and nipple waterers. The birds received their alloted feed three times a week.

Only lot A showed satisfactory performances. Both protein level and feed restriction had highly significant effects on the intensity of production and egg weight $(p<0.01)$. There was no significant interaction between these two factors. Among the different weight classes there were significant differences in egg production $(p<$ o.or) but not in egg weight. The light hens produced more eggs and consumed less feed. They also had a higher rate of mortality.

Restriction in energy and protein had different effects : Energy restriction of ro per cent diminished intensity of lay by 2.5 per cent without modifying egg weight. It severely reduced weight gain ( 16 per cent) but improved feed efficiency. A protein restriction of io per cent affected both the rate of lay and egg weight ( 4 and 2.5 per cent respectively). It also reduced body weight gain by 7 per cent without modifying feed efficiency. When both energy and protein were restricted the effects appeared to be additive; body weight gain and total weight of eggs produced were the most severely diminished. Feed efficiency was slightly improved.

These general treatment effects were not always the same in each weight class : Light hens were not sensitive to restriction in energy intake ; in fact their feed efficiency was always improved. On the other hand these birds suffered considerably from the reduced protein intake. The 
heavy hens in contrast showed a reduction of laying rate and more particulary of egg weight when energy intake was restricted but suffered very little from reduced protein intake.

The relationships between egg production and energy or protein consumption showed that the daily requirements of the J. V. I 5 breeder is of the order of $285 \mathrm{kcal}$ of ME and $\mathrm{r}_{7} \mathrm{~g}$ of protein (or $0.77 \mathrm{~g}$ of lysine or $0.64 \mathrm{~g}$ of sulfur amino-acids). Based on the different responses among weight classes it is suggested that the above recommendations should be modified according to body weight that depends on the degree of restriction during growth. Others factors such as ambient temperature should also be taken into account.

Two additional studies seem to indicate that the sulfur aminoacids requirement for maximum egg production ( 0.64 per day) is not sufficient for maximum growth of the progeny : supplemental methionine ( $0.2 \mathrm{~g}$ per day) improved the weight gain of the progeny by 5 per cent at two or four weeks of age. This improvement was no longer significant at six weeks of age. In one of these two trials the supplemental methionine also increased egg weight. Further studies are necessary to elucidate the " dietary maternal effect " of methionine. It is not known for example if this effect is specific for this dwarf strain.

In conclusion the Vedette $I . N . R . A . J V 15$ breeder hen has low nutrient requirements in terms of daily consumption but this is especially true for energy. However, to obtain maximum efficiency the body weight of this hen at onset of lay must be limited.

\section{RÉFÉRENCES BIBLIOGRAPHIQUES}

Arscott G. M., Bernier P. E., 1968. Effect of dietary protein on performance of dwarf white Leghorn layers. Poult. Sci., 47, I652 (Abstr.).

Balloun S. L., Speers G. M., I969. Protein requirements of laying hens as affected by strain. Poult. Sci., 48, II75-II88.

Bernier P. E., Arscott G. H., r960. Relative efficiency of sex-linked dwarf layers and their normal sisters, Poult. Sci., 89, 1234-1235.

BYERLY T. C., I94I. Feed and other costs of producing market eggs Maryland agric exper Stn Bull 346 I-29.

Cомвs G. F. I968. Amino acid requirements of broilers and laying hens Proc of Maryland Nutr Conf 86-96.

Damron B. L., Harms R. H., 1970. Comparison on sulphur amino acid requirements of the conventional and midget hen. Poult. Sci., 49, I379 (Abstr.).

Guillaume J., I969. Conséquences de l'introduction du gène de nanisme $d w$ sur l'utilisation alimentaire chez le poussin femelle. Ann. Biol. anim. Biochim. Biophys., 9, 369-378.

Guillaume J., 1972. Quelques particularités nutritionnelles et physiologiques du poussin nain $d w$ comparé au normal durant la croissance. Ann. Génét. Sél. anim. 4

Guillaume J., Leclerce B., Blum J.-C., I97o. Données sur les besoins alimentaires de la reproductrice naine Vedette I. N. R. A. (dw) durant les périodes de croissance et de ponte. II. Période de ponte. $X I V$ e Congrès mondial d'aviculture, Madrid, septembre I 970 .

Hutr F. B., I953. Sex-linked dwarfism in the fowl. Genetics, 28, 680.

HutT F. B., 1959. Sex-linked dwarfism in the fowl. J. Heredity, 40, 209-22 I.

JaAp G. G., Mohammadian M., I969. Sex linked dwarfism and egg production in broilers dams. Poult. Sci., 48,43.4-346.

Larbier M., Blum J.-C., Guillaume J., r971. Effets d'une déficience en lysine et méthionine sur les performances de ponte et sur la teneur en acides aminés libres du jaune d'œuf. Ann. Biol. anim. Biochim. Biophys., (sous presse).

LeclercQ B., I97o. Facteurs nutritionnels modifiant le poids de l'œuf et de ses constituants. Ann. Biol. anim. Biochim. Biophys., 10, 239-252.

Mérat P., I969. Étude d'un gène de nanisme lié au sexe chez la Poule. I. Description sommaire et performances. Ann. Génét. Sêl. anim., 1, 19-20.

Mérat P., Guillaume J., r969. Étude d'un gène de nanisme lié au sexe chez la Poule. II. Fonctionnement thyroïdien. Ann. Génét. Sél. anim., 1, I3I-I33.

Moran E. T. Jr., I969. Levels of dietary protein needed to support egg weigh and laying hen production Feedstuffs, 4i, 22-30.

Summers J. D., Rajaratnam F., Moran E. T. Jr., I97o. Is the "mini " a hypothyroidic bird? Poult. Sci., 49, I442 (Abstr.). 